\title{
Reducing exposure to COVID-19 by improving access to fever clinics: An empirical research of the Shenzhen area of China
}

\section{Qing Yong}

Wuhan University of Technology

Dinglong Liu (D1129387694@qq.com)

Wuhan Transportation Planning and Design Co., Ltd.

Guoqi Li

Southwest Jiaotong University

Wanshan Wu

Southwest Jiaotong University

Wenjie Sun

Southwest Jiaotong University

Sijing Liu

Southwest Jiaotong University

\section{Research Article}

Keywords: COVID-19, Fever clinics, SARS-CoV-2, Accessibility, Travel time

Posted Date: May 6th, 2021

DOI: https://doi.org/10.21203/rs.3.rs-453103/v1

License: (c) (1) This work is licensed under a Creative Commons Attribution 4.0 International License. Read Full License 


\section{Abstract}

\section{Background:}

The current 2019 coronavirus disease (COVID-19) pandemic is hitting citizen's life and health like never before, with its significant loss to human life and a huge economic toll. In this case, the fever clinics (FCs) were still preserved as one of the most effective control measures in China, but this work is based on experience and lacks scientific and effective guidance. Here, we use travel time to link facilities and populations at risk of COVID-19 and identify the dynamic allocation of patients' medical needs, and then propose the optimized allocation scheme of FCs $\square$

\section{Methods:}

We selected Shenzhen, China, to collect geospatial resources of epidemic communities (ECs) and FCs to determine the ECs' cumulative opportunities of visiting FCs, as well as evaluate the rationality of medical resources in current ECs. Also, we use the Location Set Covering Problem (LSCP) model to optimize the allocation of FCs and evaluate efficiency.

\section{Results:}

Firstly, we divide the current ECs into 3 groups based on travel time and cumulative opportunities of visiting FCs within 30 minutes: Low-satisfied communities (22.06\%), medium- satisfied communities (59.8\%), and high-satisfied communities (18.14\%) with 0,1-2 and no less than 3 opportunities of visiting FCs. Besides, our work proposes two allocation schemes of fever clinics through the LSCP model. Among which, selecting secondary and above hospitals as an alternative in scheme 1 , will increase the coverage rate of hospitals in high-satisfied and medium-satisfied communities from 59.8 percent to 80.88 percent. In scheme 2 , selecting primary and above hospitals as an alternative will increase the coverage rate of hospitals in medium-satisfied and large-satisfied communities to 85.29 percent, with the average travel time reducing from 22.42 minutes to 17.94 minutes.

\section{Conclusions:}

The optimized allocation scheme can achieve two objectives: a. equal access to medical services for different types of communities has improved while reducing the overutilization of high-quality medical resources. b. the travel time for medical treatment in the community has reduced, thus improving medical accessibility. On this basis, during the early screening in prevention and control of the outbreak, the specific suggestions for implementation in developing and less developed countries are made.

\section{Background}

Inequitable allocation of medical resources is the most serious problem under the outbreak and will lead to higher mortality rates ${ }^{1}$. The epidemic has continued to spread in Brazil, India, Bangladesh, and Africa ${ }^{2}$, Loading [MathJax]/jax/output/CommonHTML/jax.js such as FCs more urgent and severe. 
In response to the transmission of COVID-19, China has adopted various public health interventions to control the spread of the epidemic, including case isolation and contact tracing measures, intensive intracity and intercity traffic restriction, social distancing measures, centralized quarantine, and improvement of medical resources ${ }^{2,3}$. One of the effective measures is the establishment of a therapeutic mechanism and diversion mechanism for patients with fever or respiratory symptoms ${ }^{4}$, which aims to separate potentially infectious from non-infectious patients ${ }^{5}$ with the purpose of not only reducing the risk of cross-infection but also improving the utilization of medical resources. ${ }^{4}$. However, after the outbreak, the severity of SARS-CoV-2 pneumonia poses a great strain on critical care resources in hospitals, especially if they are not adequately staffed or resourced ${ }^{6,7}$. And the number of patients (both suspected and confirmed) greatly exceeded the number of physicians ${ }^{4}$. Now, most countries in the world face problems such as the inadequate and unbalanced supply of medical services ${ }^{8,9}$. Inadequate access to readily available healthcare has been shown to influence many health activities and outcomes, including higher mortality $1,10,11,12$, lower rates of follow-up ${ }^{13}$, increased infectious disease morbidity ${ }^{14}$, 15 . However, about $60 \%$ of suspected cases remain asymptomatic coronavirus carriers in urban communities with uneven distribution of medical resources, and risk of transmission in which is not $\operatorname{low}^{16,1718,19}$, posing a great threat to the lives of urban residents, doctors and patients ${ }^{20,21}$.

Developing equitable health systems is at the core of the United Nations Sustainable Development Goals 22, 23. However, in most countries of the world, inequalities in socio-economic status and housing conditions are often also reflected in health inequalities ${ }^{10,24,25}$. To prevent, detect, and respond to the pandemic, we require access to timely and high-quality healthcare from strong and resilient health systems $26,27,28$. Among them: improving the accessibility of health facilities, quickly connecting patients with the community health system ${ }^{15}$, and ensuring equal access to treatment for all is the key to winning this battle.

The study was conducted in Shenzhen (Fig. 1), Guangdong Province, where the first confirmed case occurred on January 19 , and the number of confirmed cases increased rapidly daily, reaching a peak on January 31. During that time, the ECs were mainly concentrated in the Nanshan District, Futian District, and Longgang District of Shenzhen, with a relatively high incidence of epidemics in the central urban areas. In the latter week, the epidemic gradually spread to the suburbs, with marked geographical differences in the confirmed cases. Finally, with the implementation of travel restrictions and border control measures, the condition for COVID-19 improved slightly and there were no new confirmed cases in the city as of 18 February. Shenzhen has only one designated hospital (The third people's hospital of Shenzhen), which can provide up to 500 beds in specific wards, as well as 49 FCs so far. Shenzhen, as the largest migrant city and special economic zone (SEZ) in China, is characterized by a high rate of population density and population movement. To prevent another outbreak, based on data availability, this paper investigates the dynamics of the medical accessibility of Shenzhen residents one week after the peak period. 
In this study, in order to truly reflect the variability in the medical accessibility under different travel modes for residents in Shenzhen, we used Baidu map real-time navigation data instead of road network calculation theory $29,30,31$ to obtain the travel time and define health facility access. What's more, travel time can be used to define accessibility in this paper due to it not only directly determines the risks of virus infection but also a key indicator in related predictions studies, such as H7N9 Avian Influenza ${ }^{31}$, African Malaria ${ }^{32}$, African Food security ${ }^{33}$. Next, accessibility measurements have been widely applied to many fields including resident commuting, public resource allocation, and urban management among the elderly and low-income groups ${ }^{34,35,36,37}$, but few studies have addressed the optimal allocation of fever clinics. For these reasons, we first analyzed the two travel modes for visiting FCs in Shenzhen, driving, and non-driving mode, and compared the differences in the availability of it. Then, we further measure variability in visiting FCs by using travel time to link facilities and populations at risk of COVID-19 and illustrate the rationality of the location of FCs. Finally, we built the Location Set Covering Problem (LSCP) based on the accessibility and cumulative opportunities of visiting FCs to balance the rational allocation of medical resources, and proposed two alternative dynamic adjustment schemes for FCs.

\section{Methods}

For our analyses, we used two data sources and two analysis methods: (1) Data of COVID-19 situation and medical resource;(2) Baidu navigation data $\mathbb{\bigotimes}(3)$ The calculation of cumulative opportunities of

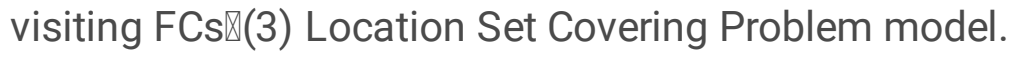

Data 1: The number of FCs and beds in designated hospitals, and the situation of the COVID-19 epidemic.

Based on the data of COVID-19 patients and medical resources publicly released by the Shenzhen Health Commission (http://wjw.sz.gov.cn/) as of February 29, 2020, including the daily-added confirmed cases (Fig. S1), the released ECs, 49 FCs, and 1 designated hospital, we identify the location of ECs and FCs using Baidu maps. This paper selects epidemic data one week after the peak of the epidemic including the number of newly confirmed cases and newly added ECs (Fig.S2), and the spatial distribution maps of ECs, FCs(Fig.S3). Furthermore, the calculation of available beds is based on the standard that designated hospitals can provide up to 500 beds in special wards. The attribute data of FCs mainly includes name, address, type, level of the hospital, and so on.

The ECs are mainly distributed in Nanshan (48), Futian (41), Longgang (37), and Baoan District (29), accounting for $76 \%$. In terms of the level of epidemic hospitals, there are 10 secondary hospitals and 35 tertiary hospitals, accounting for $20 \%$ and $71.4 \%$ respectively, are mainly concerned at Longgang District, Baoan District, Futian District, and Nanshan District.

\section{Data 2: Baidu navigation data}

To truly represent the travel time to health facilities, based on the origin/destination(O/D) point of 204 Ercs and N Frcs we arcumired the travel time of visiting FCs in three modes of walking, public Loading [MathJax]/jax/output/CommonHTML/jax.js 
transportation and driving using real-time navigation data obtained from the Baidu Map API (http://lbsyun.Baidu.com/). In accordance with the principle of visiting the nearest FCs, we compute the shortest time to visit FCs under driving mode and non-driving mode combining walking and public transportation (Fig.S4). In addition, we evaluated the transportation accessibility of the two modes.

Methods S1: The calculation of cumulative opportunities for visiting FCs.

This paper generates the OD time matrix Tij $\backslash$ invalidcharacter $204 \times n \backslash$ invalidcharacterbased on the travel time tij between regions. The threshold range for travel time is below $15,15-30,30-45$, and above 45. Observing whether the travel time tijsatisfies the threshold value to generate the cumulative opportunities of visiting FCs, the formula is as follows:

$$
\begin{gathered}
C_{i j k}=\sum_{j \in V} X_{i j k}, \quad \forall i \in U, \quad k \in\{1,2\} \\
X_{i j k} \in\{0,1\}, \quad \forall i \in U, j \in V, k \in\{1,2\} \\
t_{i j} \in T_{i j}, \quad \forall i \in U, j \in V
\end{gathered}
$$

Where

$U=$ A set of 204 urban ECs, community $i \in U$;

$V=$ A set of FCs in Shenzhen, fever clinic $j \in V$;

$k=$ Two modes of visiting FCs for community residents, which takes a value of 1 , if visiting in non-driving mode, otherwise it is 2 ;

$X_{i j}=\mathrm{A} 0-1$ variable, which assumes a value of 1 if the travel time from a community $i$ to hospital $j$ in $k$ mode meets the threshold range, otherwise, it is 0 .

$C_{i j k}=$ Cumulative opportunities of visiting FCs in mode $k$ within a community $i$, reflecting the perfect degree of the medical facilities around the community.

\section{Methods S2: Location Set Covering Problem model}

To improve the utilization efficiency of high-quality medical resources and shorten the gap in visiting opportunities and transportation accessibility between different ECs, this paper employs the Location Set Covering Problem (LSCP) model to optimize the allocation of FCs 38,39 , which is as follows: 


$$
\begin{aligned}
& \text { Min } \sum_{\mathrm{jev}} A_{\mathrm{j}} \\
& \text { s.t. } \sum_{i \in U} X_{i j} \leq A_{j} * W, \quad \forall j \in V \\
& \sum_{j \in V} X_{i j} \geq 1, \quad \forall i \in U \\
& A_{j} \in\{0,1\}, \quad \forall j \in V \\
& X_{i j} \in\{0,1\}, \quad \forall i \in U, j \in V
\end{aligned}
$$

Where

$W=$ Infinite positive number.

$A_{j}=\mathrm{A} 0-1$ variable, which assumes a value of 1 if a hospital $j$ is determined to be a fever clinic for a community $i$. otherwise, it is 0 .

$X_{i j}=$ A $0-1$ variable, which assumes a value of 1 if the shortest travel time from a community $i$ to a hospital $j$ exceeds 30 minutes, otherwise, it is 0 .

The objective function (Eq. (3a)) attempts to cover the maximum number of ECs with the minimum number of facilities. Eq. (3b) assumes that if the shortest time-consuming from the community to the hospitaldoes not exceed 30 minutes, the hospital is selected as a fever clinic for treatment in the community. Eq. (3c) means that each epidemic community must be covered by just one fever clinic.

\section{Results}

Measures of transportation accessibility for ECs under different transport modes.

Referring to the accessibility-based measurements proposed in Hulland and Van Wee ${ }^{40}$, this paper reckoned cumulative opportunities for visiting FCs based on the travel time of ECs ${ }^{41}$. Stemming from 2016 urban resident trip surveys in Shenzhen, more residents prefer traveling in non-driving models, accounting for $74 \%$. But it is safer and faster to drive during the outbreak of the epidemic. Therefore, taking 15 minutes, 30 minutes, and 45 minutes as a measurement index, we calculated the cumulative opportunities of visiting FCs under the two models.

Figure $2 \mathrm{a}, \mathrm{b}$ shows that within 15 minutes, $77.45 \%$ of the ECs traveling in non-driving mode could not arrive on time to the nearest FCs, while only $3.43 \%$ in driving mode could not reach. Most of these communities are located near district boundaries, such as the west of Longgang District, the east of the Yantian District, and the south of Longhua District. Figure 2b, e shows traveling in driving mode within 15 minutes is equivalent to traveling in non-driving mode within 45 minutes. Similarly, the number of inaccessible ECs and FCs is very close between the two models, accounting for $3.43 \%$ and $1.47 \%$, respectively; Still, there are 45 communities accounting for $22.06 \%$ that cannot reach the nearest hospital in the non-driving mode while people there can achieve it by driving within 30-minutes travel thresholds(Fig. 2c, d). 
On the whole, traveling in non-driving mode means more travel time, resulting in some people turning to seek treatment in non-government designated medical institutions. This way of treatment is not conducive to timely diagnosis and follow-up for patients, thereby increasing the risk of prevention and control.

Rationality evaluation of medical resources.

According to the plan of the National Health Commission of the PRC, by 2020, China will achieve a 30 minutes primary medical service circle. Bosanac ${ }^{42}$ advocated a similar criterion, so this paper investigated the cumulative opportunities of visiting FCs under the non-driving mode in ECs within 30 minutes, to explore the balance between medical resources needs and supplies in each district.

To begin with, we explored the current status of medical resources in all the districts. Figure 3a shows that the number of FCs in the first 4 districts with the severe epidemic in Shenzhen, is the opposite. Especially there are only 5 FCs in Nanshan District with the lowest average number of clinics owned by the ECs, only 0.1 , followed by Longhua and Luohu District of 0.17 and 0.19 . Then the significant contradiction and imbalance between supply and demand of medical resource allocation are further revealed based on the cumulative opportunities of visiting FCs. Figure $3 \mathrm{~b}$ indicates that 45 communities with 0 opportunities of visiting FCs are mainly distributed in Nanshan, Longgang, Longhua, Baoan, and Guangming Districts. In mainland China, FCs affiliated with the emergency departments (EDS),the setting it is related to hospital service capacity (standard). Of FCs released by the Shenzhen government, the tertiary hospitals secondary, primary, and below hospitals are accounted for $71.4 \%, 20.4 \%$, and $8.2 \%$ respectively. Moreover, about $62 \%$ of FCs in secondary and above hospitals are located in Futian, Longgang, and Baoan Districts. Thanks to that the initial site selection scheme was constrained by the service capacity and location of graded hospitals, it was difficult to achieve equitable access to medical treatment for residents in different communities, thus the location of FCs was not reasonable.

Next, based on the cumulative opportunities of visiting FCs within the ECs in 30 minutes, 3 social groups can be captured (Fig. 4). Among them, the community with 0 opportunities of visiting in 30 minutes is a "low-satisfied community" (22.06\%), scattered in every district, the average travel time for them is 42 minutes. In communities with no greater than 3 cumulative opportunities of visiting FCs, "mediumsatisfied communities" account for $59.8 \%$ with 20 minutes of average travel time. High-satisfied communities are concentrated in Futian, Baoshan, and Luohu District, accounting for $18.14 \%$, with 4 or more cumulative opportunities of visiting FCs and the 15 minutes of average travel time. This illustrates that $40.2 \%$ of FCs need to be further optimized to improve medical accessibility and reduce transmission of COVID-19.

\section{Optimized allocation of FCs and effect evaluation}

Considering that there are more than 218 hospitals and 661 community health centers in Shenzhen that have not been included as FCs for the coronavirus epidemic, combined with that current FCs need to Loading [MathJax]/jax/output/CommonHTML/jax.js he outbreak is stable, it is practical to dynamically adjust the 
distribution of the fever clinics. The LSCP (Location Set Covering Problem) model ${ }^{38,43}$ which attempts to locate a minimum number of servers to cover all demand nodes within the time standard, can achieve the goal of optimizing the rational and balanced use of medical resources and medical equity. Accordingly, we propose two optimization schemes: (i) adding secondary and above hospital as alternatives, (ii) adding primary and above hospital as alternatives. In order to make maximum utilization of medical resources, if the new scheme cannot completely cover the current low-satisfied ECs during the dynamic adjustment process, we will set community hospitals to provide medical services for it. Afterward, we calculated the minimum arrival time-based on the Baidu map API, and finally build the LSCP model to obtain two allocation schemes of FCs.

Table 1 shows that compared with 49 FCs before adjustment, the number of FCs in schemes 1 and 2 have become 32 and 35, respectively. Among them: on the basis of guarantee accessibility, the number of tertiary and above hospitals has decreased by 22 and 18 respectively, which has played an active role in getting medical order to normal.

Table 1

Allocation schemes of FCs and comparison of changes in coverage levels

\begin{tabular}{|c|c|c|c|c|}
\hline & Items & Current scheme & Scheme 1 & Scheme 2 \\
\hline \multirow[t]{5}{*}{ Hospitals levels with FCs } & Tertiary & 35 & 17 & 13 \\
\hline & Secondary & 10 & 14 & 10 \\
\hline & Primary & 2 & 1 & 12 \\
\hline & Non-level & 2 & 0 & 0 \\
\hline & Community hospitals & 0 & 28 & 19 \\
\hline \multirow[t]{2}{*}{ Low-satisfied community } & number & 45 & 28 & 19 \\
\hline & travel time & 37 & 42 & 38 \\
\hline \multirow[t]{2}{*}{ Medium-satisfied community } & number & 122 & 165 & 174 \\
\hline & travel time & 19 & 20 & 16 \\
\hline \multirow[t]{2}{*}{ high-satisfied community } & number & 37 & 11 & 11 \\
\hline & travel time & 16 & 15 & 14 \\
\hline
\end{tabular}

Specifically, there are 122 medium-satisfied communities in the current scheme, with a coverage rate of only $59.8 \%$ and the average travel time is 22.42 minutes (Fig. 4a). After the optimization that retaining 21 original FCs and adding another 11 secondary and above hospitals in scheme 1 (Fig. 4b), the coverage rate of community medium- and high-satisfied hospitals are up to $80.88 \%$, and the average time of going to the FCs is 22.75 minutes; In the scheme 2(Fig. 4c), only 15 original FCs are reserved while 20 hospitals are newly added. As a result, the coverage rate of the medium- and high- satisfied community reach 
above hospitals as alternatives, scheme 1 can improve the fairness of visiting in ECs, transferring the high- and low-satisfied communities to medium-satisfied communities; By choosing hospital at a low level such as primary and above hospitals, scheme 2 can achieve a higher coverage rate, reduce the travel time and improve accessibility.

In addition, for the ECs that can't be covered, 28 and 19 community hospitals are required to provide services, suggesting that primary medical institutions should play a positive role in confronting the virus. Wuhan, the center of the epidemic in China, has adopted similar measures to achieve rapid screening of patients with fever or respiratory symptoms.

Apropos of the COVID-19, the allocation of medical resources must consider the balance and difference between different regions. Inequality in health care comes at the expense of individuals and society, and this will be reflected in inequality in the outcome of the epidemic ${ }^{44}$. Figure $4 a$ demonstrates that highsatisfied communities are mainly distributed in Bao'an, Futian, and Luohu Districts, which are in the core urban areas of Shenzhen. In contrast, low-satisfied communities are usually located in borders of districts lacking medical resources. In the meantime, the migrant worker after the lunar new year in 2019 in Shenzhen is the highest, accounting for $4.54 \%$, and it will experience large population movements. So that may result in the recurrent spread of the COVID-19 if the medical accessibility of suspected cases is not effectively improved. Through the dynamic adjustment of FCs, the gap between medical accessibility in high-satisfied and low-satisfied communities has been greatly reduced. Supported by the active involvement of primary medical institutions, the ECs that cannot be covered within 30 minutes have been effectively covered, which is necessary for the disadvantaged communities in the junction of the districts. During the rapid spread of the epidemic, many cities have made an immediate reaction and quickly provide a large number of fever clinics (for example 392 FCs in Chengdu and 426 FCs in Chongqing). In addition, these cities are dynamically optimizing and moderately reducing the FCs according to the situation of the epidemic (for example, the number of FCs in Beijing has been adjusted from 89 to 76).

\section{Discussion}

Through this study, we show that if using the non-traveling mode, there are more than $75 \%$ of residents in Shenzhen have lower access to treatment. According to the statistical results, 45 Low-satisfied communities $(22.06 \%)$ were unable to reach the nearest fever clinic during the 30-min in non-driving mode, mainly in Nanshan District (9), Longhua District (9), and Longgang District (9).In contrast, about $60 \%$ of the FCs in Shenzhen are located in Futian, Longgang, and Baoan districts. On the whole, there is an unbalanced distribution of ECs and fever clinic resources in various districts. for example, Nanshan District has the largest number of confirmed cases while the average percentage of FCs in the epidemic community is the lowest, only 0.1 , followed by Longhua District (0.17) and Luohu District (0.19); In addition, the data show that $91.8 \%$ of FCsof secondary and above hospitals, mainly concentrated in Futian District, Longgang District and Baoan District. As a result, these facilities can not achieve equitable access to medical treatment for each district and county, and even more difficult to address the issue of

Loading [MathJax]/jax/output/CommonHTML/jax.js nity level.

Page 9/18 
In order to overcome the above problems about unbalanced resource allocation, the LSCP model was built to realize the dynamics of FCs in Shenzhen, and two effective solutions were proposed based on the selection of different levels of alternative FCs. To be more exact, choosing secondary and above hospitals as alternatives in scheme 1 can reduce the difference between different community groups and effectively increase the coverage of ECs to $80.88 \%$, but it can't effectively reduce the average time available for residents to visit FCs. However, selecting primary and above hospitals as alternatives can decrease the average travel time by 17.94 minutes while reducing the differences in community groups, improving medical accessibility. In addition, the proportion of tertiary hospitals in scheme 2 was reduced from 71.43 percent to 24.07 percent, reducing the overutilization of high-quality medical resources. Therefore, in the context of the current epidemic and other future public health crises, a community-wide, well-resourced, health-equitable primary health care system is not only a central policy of the national health system and emergency response ${ }^{4529}$, but also a basic safety guarantee for the sustainable development of people's lives and health.

With the widespread of an epidemic all over the world, there is an increasingly urgent health care response to the epidemic in various countries, particularly in developing countries with low efficiency of health systems. For example, in densely populated countries with inadequate medical resources, such as Brazil, India, and Bangladesh, the epidemic has spread to low-income groups in villages, shanty towns and favelas, and slums who work in dense, unplanned areas and cannot be provided with basic public facilities and services, including public health systems. Thus, grass-root people are fighting with chaos, hunger, and death during the outbreak. We conclude that, given the inadequate and unbalanced allocation of medical resources in most countries as well as lacking scientific and effective guidance for the allocation of FCs, if the FCs are allocated based on experience alone, it may cause the second wave.

Therefore, based on the optimal allocation of FCs, several measures are proposed for developing and less developed countries with severe epidemic:(1)Firstly, according to the susceptible population (e.g. the elderly, low-income groups) or where the susceptible population is gathering, the current hospitals (including public hospitals and private hospitals) are selected as alternative hospitals based on the principle of visiting the nearest FCs. Then, based on the principle of maximum coverage, the FCs are distributed for rapid screening and classification of patients during early-stage.(2) Temporary mobile FCs should be established as soon as possible in areas with scarce resources and high risk, reducing the difference in medical accessibility between different communities and shorting the travel time for patients.ख3) The government should dynamically adjust and balance the allocation of FCs according to the direction of the spread of the epidemic and new changes in communities and areas. $₫ 4$ \اn terms of setting up FCs, the basic medical resources such as community hospitals and private clinics should be utilized and transformed as much as possible, so as to reduce the excessive occupation of high-quality medical resources and restore normal medical order as soon as possible. (5) Setting up temporary testing laboratories in the public basic medical institution with FCs or combining five or more individuals samples for mixing tests if necessary, is essential for rapid screening. (6) Lastly, governments should 
urgently raise funds and establish FCs to ensure that early screening and quarantine measures are effectively implemented to prevent the further spread of the epidemic.

\section{Limitations}

Still, several limitations need to be mentioned. One limitation is that Shenzhen has not yet announced the number of confirmed and suspected cases in the epidemic community which can't allow us to track the severe situation of the epidemic and determine the supply and demand matching relationship between patients (within the hospital's service area) and hospital beds (or medical workers). So that we can't observe the contradiction between the supply and demand and can't explore plans for the deployment of new resources. Another limitation is the inadequate description of the accessibility evaluation index of visiting FCs in the epidemic community. We only considered that long-term travel will increase the risk of infection, but did not fully consider factors such as transfers times, the density of transfer hubs, and the patient's attributes (income, age, and degree of illness, etc.), which will have a certain impact on the risk of exposure to the virus and behavior of patients.

\section{Abbreviations}

COVID-19: 2019 coronavirus disease; FCs: Fever clinics; ECs: Epidemic communities; LSCP: Location Set Covering Problem

\section{Declarations}

\section{Ethics approval and consent to participate}

Not applicable

\section{Consent for publication}

Not applicable

\section{Availability of data and materials}

The datasets used and/or analyzed during the current study are publicly available from the Shenzhen Health Commission(http://wjw.sz.gov.cn/yqxx/index.html) and Baidu Map API (http://lbsyun.Baidu.com/).

\section{Competing Interest}

The authors declare that they have no competing interests

\section{Funding}


National Natural Science Foundation of China (approval numbers 71603219 and 41501123) and National Engineering Laboratory of the National Transportation Comprehensive Big Data Application Technology Program (approval number CTBDAT201909) for the collection, analysis, and interpretation of data and in writing the manuscript

\section{Authors' contributions}

Qing Yong[Conceptualization, Methodology, Formal analysis, Investigation, Data Curation, Writingoriginal draft, Writing-review \& editing, Visualization, Project administration. Dinglong Liu: Methodology, Formal analysis, Investigation, Data Curation, Writing-original draft, Writing-review \& editing, Visualization, Project administration. Guoqi Li: Conceptualization, Methodology, Formal analysis, Investigation, Writingoriginal draft, Writing-review \& editing, Supervision, Project administration, Funding acquisition. Wanshan Wu: Writing-original draft, Writing-review \& editing. Wenjie Sun: Methodology, Software. Sijing Liu: Conceptualization, Methodology

\section{Acknowledgments}

We thank Professor Fahui Wang for his guidance on the structure of the paper, as well as the National Natural Science Foundation of China (Grants 71603219 and 41501123) and the National Engineering Laboratory of Integrated Transportation Big Data Application Technology Program (Grant CTBDAT201909) for their support.

\section{References}

1. Wang Z, Tang K. Combating COVID-19: health equity matters. Nat Med 26, 458 (2020)

2. He Z. What further should be done to control COVID-19 outbreaks in addition to cases isolation and contact tracing measures? BMC Medicine 18, 80 (2020)

3. Pan A, et al. Association of Public Health Interventions With the Epidemiology of the COVID-19 Outbreak in Wuhan, China. JAMA, (2020)

4. Wang J, et al. Separate Fever Clinics Prevent the Spread of COVID-19 and Offload Emergency Resources: Analysis from a large tertiary hospital in China. medRxiv.https://doi.org/10.1101/2020.04.03.20051813 (2020)

5. Hu Z, et al. Clinical Characteristics of 24 Asymptomatic Infections with COVID-19 Screened among Close Contacts in Nanjing, China. (2020)

6. Yang $X$, et al. Clinical course and outcomes of critically ill patients with SARS-CoV-2 pneumonia in Wuhan, China: a single-centered, retrospective, observational study. The Lancet Respiratory Medicine, (2020)

7. COVID-19: too little, too late? The Lancet 395.10.1016/s0140-6736(20)30522-5 (2020)

8. Kyu HH, et al. Global, regional, and national burden of tuberculosis, 1990-2016: results from the Glohal Rurden of Diseases Iniuries and Risk Factors 2016 Study. Lancet Infectious Diseases 18, Loading [MathJax]/jax/output/CommonHTML/jax.js 
1329-1349 (2018)

9. X. Zhao, J. Fan. Data portrait of 1,183 assistants: not the weak, but you and me. RUC News, (2020)

10. Dorn Av, Cooney RE, Sabin ML. COVID-19 exacerbating inequalities in the US The Lancet 395, 1243$1244(2020)$

11. Syed ST, Gerber BS, Sharp LK. Traveling towards disease: transportation barriers to health care access. J Community Health 38, 976-99323543372 (2013)

12. Wagenaar $\mathrm{BH}$, et al. Developing a representative community health survey sampling frame using open-source remote satellite imagery in Mozambique. International Journal of Health Geographics 17, 37 (2018)

13. Kelly C, Hulme C, Farragher TM, Clarke G. Are differences in travel time or distance to healthcare for adults in global north countries associated with an impact on health outcomes? A systematic review. BMJ Open 6, 013059 (2016)

14. O'Meara WP, Noor A, Gatakaa H, Tsofa B, McKenzie FE, Marsh K. The impact of primary health care on malaria morbidity-defining access by disease burden. Trop Med Int Health 14, 29-3519121148 (2009)

15. Hulland EN, et al. Travel time to health facilities in areas of outbreak potential: maps for guiding local preparedness and response. BMC Med 17, 23231888667 (2019)

16. Wang C, et al. Evolving Epidemiology and Impact of Non-pharmaceutical Interventions on the Outbreak of Coronavirus Disease 2019 in Wuhan, China. medRxiv.10.1101/2020.03.03.20030593 (2020)

17. Mizumoto K, Kagaya K, Zarebski AE, Chowell G. Estimating the asymptomatic proportion of coronavirus disease 2019 (COVID-19) cases on board the Diamond Princess cruise ship, Yokohama, Japan, 2020. Eurosurveillance 25, 2000180 (2020)

18. Qiu J. Covert coronavirus infections could be seeding new outbreaks. nature news. nature news. https://www.nature.com/articles/d41586-020-00822-x (2020)

19. Woelfel R, et al. Clinical presentation and virological assessment of hospitalized cases of coronavirus disease 2019 in a travel-associated transmission cluster. medRxiv.10.1101/2020.03.05.20030502 (2020)

20. Wu Z, McGoogan JM. Characteristics of and Important Lessons From the Coronavirus Disease 2019 (COVID-19) Outbreak in China: Summary of a Report of 72314 Cases From the Chinese Center for Disease Control and Prevention. JAMA 323, 1239-1242 (2020)

21. Webster P. Canada and COVID-19: learning from SARS. The Lancet 395, 936-937 (2020)

22. Council S. LexisNexis http://china.lexiscn.com/law/law-english-1-2961294-T.html. (25 October 2016)

23. Marmot M, Bell R. Epidemiology 29, 5-7 (2018)

24. Emilie Alirol, Laurent Getaz, Beat Stoll, François Chappuis, Loutan L. Urbanisation and infectious diseases in a globalised world. The Lancet Infectious Diseases.10.1016/S1473-3099(10)70223-1 (2n11)

Loading [MathJax]/jax/output/CommonHTML/jax.js

Page 13/18 
25. Raifman M, Raifman J. Disparities in the Population at Risk of Severe Illness From COVID-19 by Race/Ethnicity and Income. American Journal of Preventive Medicine, (2020)

26. Heymann DL, et al. Global health security: the wider lessons from the west African Ebola virus disease epidemic. The Lancet 385, 1884-1901 (2015)

27. Kruk ME, Myers M, Varpilah ST, Dahn B. What is a resilient health system? Lessons from Ebola. The Lancet 385, 1910-1912 (2015)

28. Peters D, Garg A, Bloom G, Walker D, Brieger WR, Rahman MH. Poverty and Access to Health Care in Developing Countries. Annals of the New York Academy of Sciences 1136, 161-171 (2008)

29. M. Chen, Y. Chen, X. Wang H, Tan FL. Spatial Difference of Transit-Based Accessibility to Hospitals by Regions Using Spatially Adjusted ANOVA. International journal of environmental research and public health 16, 19-23 (2019)

30. Weiss DJ, et al. A global map of travel time to cities to assess inequalities in accessibility in 2015 . Nature 553, 333-336 (2018)

31. Gilbert $M$, et al. Predicting the risk of avian influenza A H7N9 infection in live-poultry markets across Asia. Nature Communications 5, 4116 (2014)

32. Bhatt $\mathrm{S}$, et al. The effect of malaria control on Plasmodium falciparum in Africa between 2000 and 2015. Nature 526, 207-211.10.1038/nature15535 (2015)

33. Frelat $\mathrm{R}$, et al. Drivers of household food availability in sub-Saharan Africa based on big data from small farms. 113, 458-463 (2016)

34. Ouyang L, Yin Z, Wang D. Visualizing disparities in park access for the elderly in Shanghai, China. Environment Planning A: Economy Space 52, 260-265 (2020)

35. Talen E. School, community, and spatial equity: An empirical investigation of access to elementary schools in West Virginia. Annals of the Association of American Geographers 91, 465-486 (2001)

36. Tsou K-W, Hung Y-T, Chang Y-L. An accessibility-based integrated measure of relative spatial equity in urban public facilities. Cities 22, 424-435 (2005)

37. Jin M, Liu L, Tong D, Gong Y, Liu Y. Evaluating the spatial accessibility and distribution balance of multi-level medical service facilities. International journal of environmental research public health 16 , 1150 (2019)

38. Toregas C, Swain R, ReVelle C, Bergman L. The location of emergency service facilities. Operations research 19, 1363-1373 (1971)

39. Farahani RZ, Asgari N, Heidari N, Hosseininia M, Goh M. Covering problems in facility location: A review. Computers \& Industrial Engineering 62, 368-407 (2012)

40. Van Wee B, Geurs K. Discussing equity and social exclusion in accessibility evaluations. European journal of transport and infrastructure research 11, (2011)

41. Chen M, Chen Y, Wang X, Tan H, Luo F. Spatial Difference of Transit-Based Accessibility to Hospitals by Regions Using Spatially Adjusted ANOVA. International journal of environmental research and nuhlir health $161922(2019)$

Loading [MathJax]/jax/output/CommonHTML/jax.js

Page 14/18 
42. Bosanac EM, Parkinson RC, Hall DS. Geographic access to hospital care: a 30-minute travel time standard. Medical Care 14, 616-624 (1976)

43. Hakimi SL. Optimum locations of switching centers and the absolute centers and medians of a graph. Operations research 12, 450-459 (1964)

44. Wang F. Measurement, optimization, and impact of health care accessibility: a methodological review. Annals of the Association of American Geographers 102, 1104-1112 (2012)

45. Roder-DeWan S. Health system quality in the time of COVID-19. Lancet Glob Health 2020, (2020)

\section{Figures}
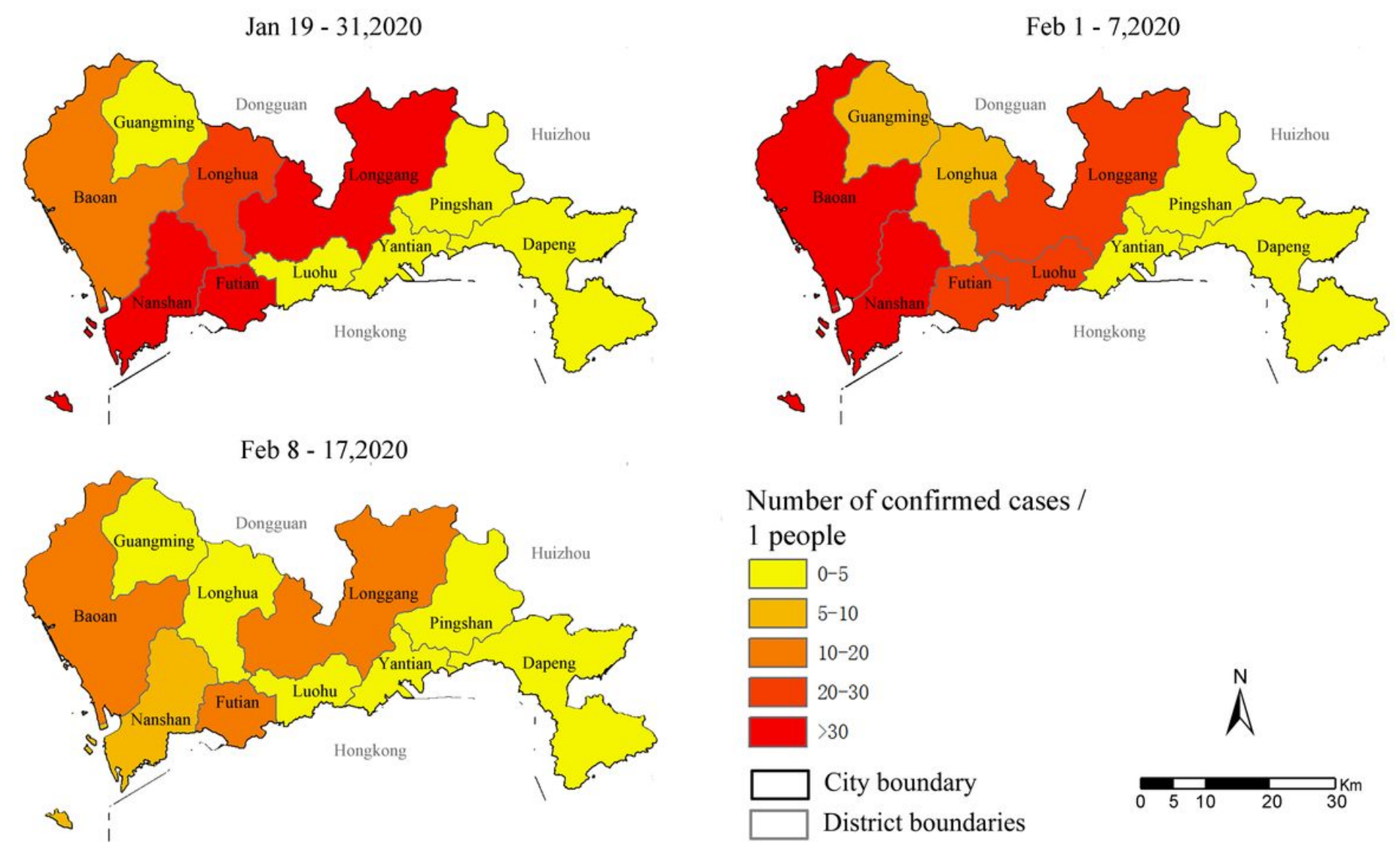

Figure 1

The Geographic Distribution of Daily Rates of COVID-19 Cases Across the 3 Periods in Shenzhen, China. The daily rate of cases is expressed as the number of confirmed cases per day per 1 person, grouped by each of the 10 districts of the city of Shenzhen. Note: The designations employed and the presentation of the material on this map do not imply the expression of any opinion whatsoever on the part of Research Square concerning the legal status of any country, territory, city or area or of its authorities, or concerning the delimitation of its frontiers or boundaries. This map has been provided by the authors. 

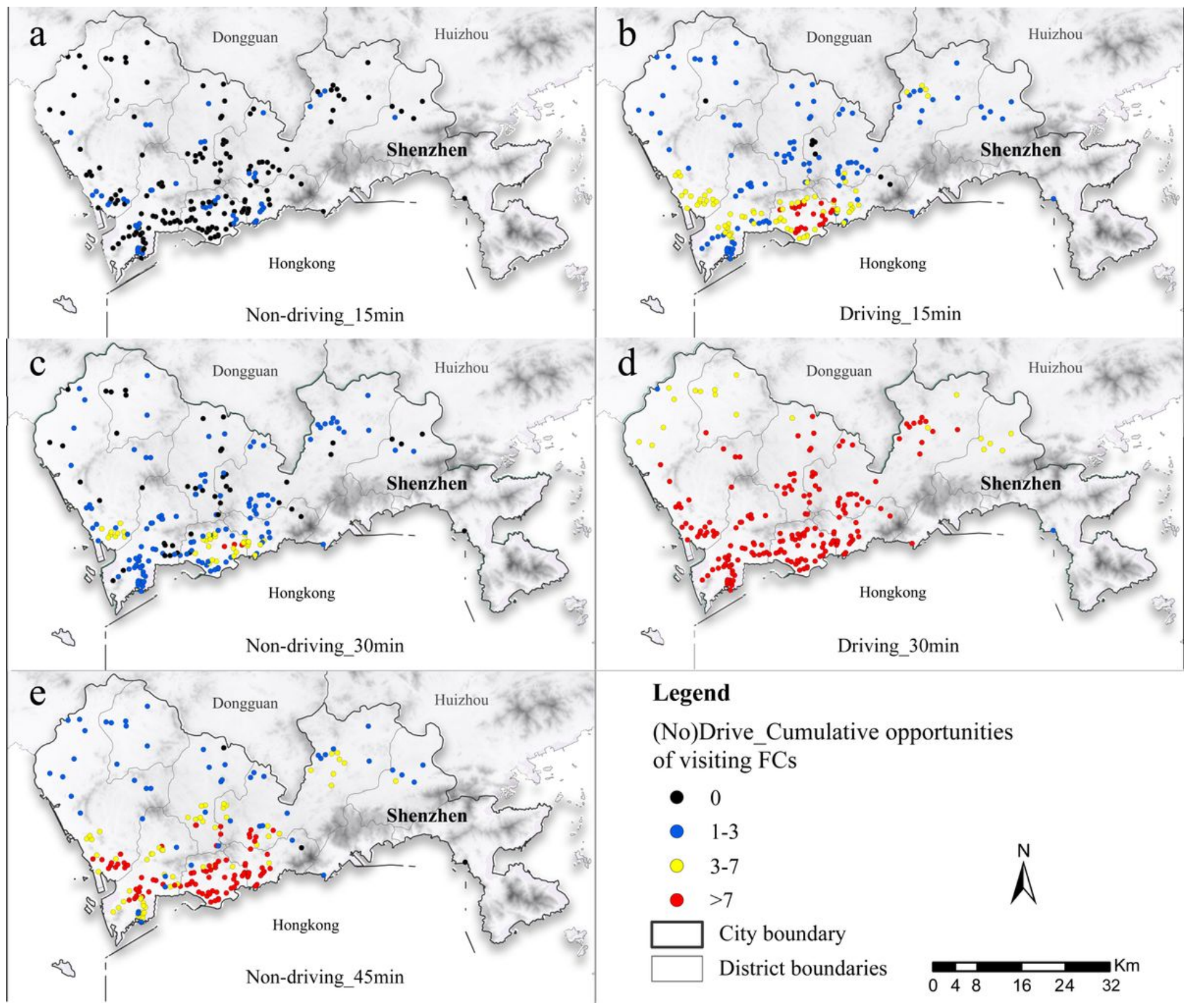

\section{Legend}

(No)Drive_Cumulative opportunities of visiting $\mathrm{FCs}$
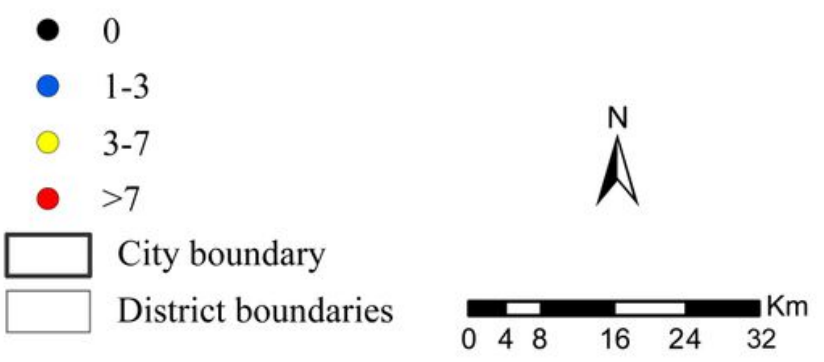

Figure 2

Number of FCs accessible by ECs under different travel modes and time constraints. a, b respectively represents the number of accessible FCs under the non-driving and driving modes with a travel time limit of 15 minutes (inclusive). c, d respectively demonstrates the number of accessible FCs under non-driving and driving modes with a travel time limit of 30 minutes (inclusive). e manifests the number of accessible FCs under non-driving modes with a travel time limit of 45 minutes (inclusive). Note: The designations employed and the presentation of the material on this map do not imply the expression of any opinion whatsoever on the part of Research Square concerning the legal status of any country, territory, city or area or of its authorities, or concerning the delimitation of its frontiers or boundaries. This map has been provided by the authors. 


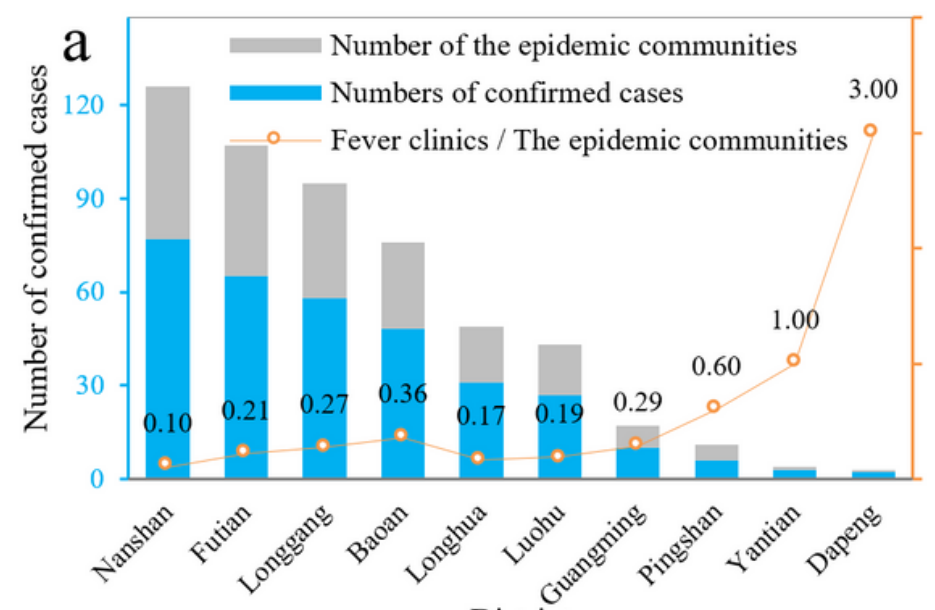

Districts

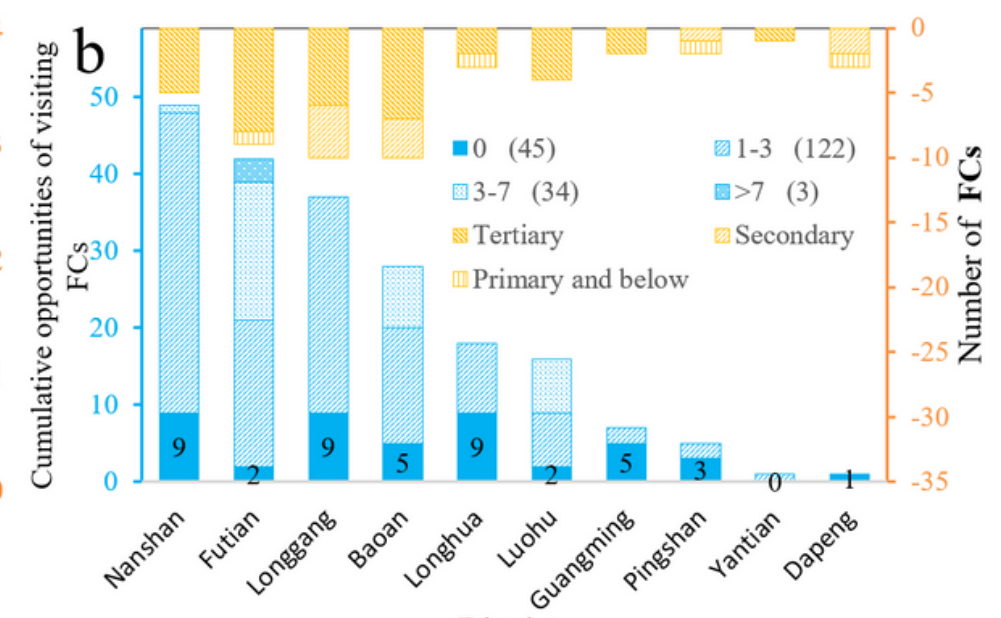

Districts

Figure 3

a Numbers of confirmed cases, numbers of the ECs and numbers of FCs in Shenzhen (Data source: Shenzhen Municipal Health Commission.); b Cumulative opportunities of visiting FCs based on 30minutes travel thresholds and number of FCs in Shenzhen (summarized by hospital levels) (Data source: Baidu map navigation data).

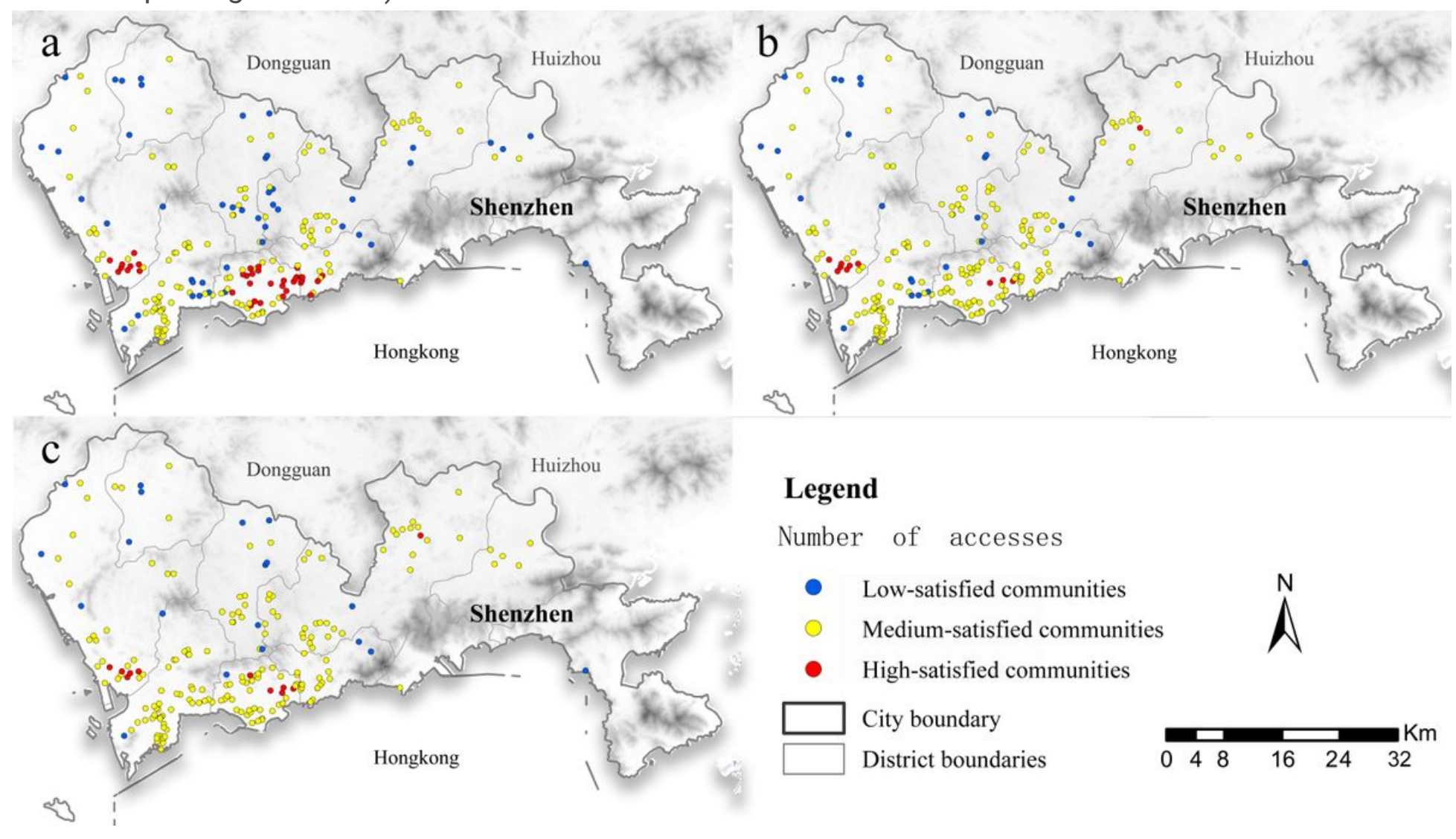

Figure 4

a. The current scheme: based on community groups with $49 \mathrm{FCs}$, there are 122 medium-satisfied communities, 45 low-satisfied communities, and 37 high-satisfied communities. b. Scheme 1: based on Loading [MathJax]/jax/output/CommonHTML/jax.js 
community groups with 32 secondary and above hospitals as the FCs $₫$ there are 165 medium-satisfied communities, 28 low-satisfied communities, and 11 high-satisfied communities; c. Scheme 2: based on community groups with 35 primary and above hospitals as the FCs, there are 174 medium-satisfied communities, 19 low-satisfied communities, and 11 high-satisfied communities. Note: The designations employed and the presentation of the material on this map do not imply the expression of any opinion whatsoever on the part of Research Square concerning the legal status of any country, territory, city or area or of its authorities, or concerning the delimitation of its frontiers or boundaries. This map has been provided by the authors.

\section{Supplementary Files}

This is a list of supplementary files associated with this preprint. Click to download.

- SupplementaryInformation.docx 